\title{
Gap Phenomenon of an Abstract Willmore Type Functional of Hypersurface in Unit Sphere
}

\author{
Yanqi Zhu, Jin Liu, and Guohua Wu \\ College of Information System and Management, National University of Defense Technology, Changsha, Hunan 410073, China \\ Correspondence should be addressed to Jin Liu; liujin229234@163.com
}

Received 18 April 2014; Accepted 26 May 2014; Published 5 June 2014

Academic Editor: Luc Vrancken

Copyright ( 2014 Yanqi Zhu et al. This is an open access article distributed under the Creative Commons Attribution License, which permits unrestricted use, distribution, and reproduction in any medium, provided the original work is properly cited.

For an $n$-dimensional hypersurface in unit sphere, we introduce an abstract Willmore type called $W_{(n, F)}$-Willmore functional, which generalizes the well-known classic Willmore functional. Its critical point is called the $W_{(n, F)}$-Willmore hypersurface, for which the variational equation and Simons' type integral equalities are obtained. Moreover, we construct a few examples of $W_{(n, F)}-$ Willmore hypersurface and give a gap phenomenon characterization by use of our integral formula.

\section{Introduction}

Let $\varphi: M^{n} \rightarrow S^{n+1}(1)$ be an $n$-dimensional compact without boundary hypersurface in unit sphere. Choose an orthonormal frames field $\left\{e_{1}, \ldots, e_{n}, e_{n+1}\right\}$ along $M$ such that $\left\{e_{1}, \ldots, e_{n}\right\}$ are tangent to $M$ and $\left\{e_{n+1}\right\}$ is normal to $M$. Their dual frames are $\left\{\theta^{1}, \ldots, \theta^{n}\right\}$ and $\left\{\theta^{n+1}\right\}$, respectively; obviously, $\theta^{n+1}=0$ when it is restricted over $M$. Let $h$ denote the second fundamental form of the immersion $\varphi: M \rightarrow$ $S^{n+1}(1)$. Write

$$
h=h_{i j} \theta^{i} \otimes \theta^{j}
$$

and define

$$
\begin{array}{ll}
H=\frac{1}{n} \sum_{i=1}^{n} h_{i i}, & S=\sum_{i j}\left(h_{i j}\right)^{2}, \\
\rho=S-n H^{2}, & P_{k}=\operatorname{tr}\left(h^{k}\right) .
\end{array}
$$

One calls $H, S, P_{k}$ to be mean curvature, square of the length, and $k$ th power polynomial of second fundamental form, respectively. It is well known that hypersurface $\varphi: M^{n} \rightarrow$ $S^{n+1}(1)$ is minimal when $H \equiv 0$ and is totally geodesic when $S \equiv 0$. For invariant $\rho$, it is obvious that $\rho(Q) \geq 0$ for all $Q \in M, \rho(Q)=0$ if and only if $Q$ is an umbilical point of $M$, and $0 \leq \rho(Q) \leq C$ for all $Q \in M$ and some positive constant $C$ since $M$ is compact.
In differential geometry, there is a famous classical Willmore functional of hypersurface which is defined as

$$
W_{(n, n / 2)}(\varphi)=\int_{M} \rho^{n / 2} d v
$$

See [1-4] for the details. It is well known that this functional is invariant under conformal transformation. Its critical point is called classical Willmore or $W_{(n, n / 2)}$-Willmore hypersurface. The famous Willmore conjecture says that $W_{(2,1)}(\varphi) \geq 4 \pi^{2}$ holds for all immersed tori $\varphi: M \rightarrow S^{3}$; recently, it has been completely solved by Marques and Neves in article [5] using Min-Max theory for two dimensional case, but higher dimensional case is still an open problem. When $n \geq 3$, the functional $W_{(n, n / 2)}(\varphi)$ was studied extensively in [6-11].

Due to the importance of Willmore conjecture, many geometric experts generalized the classic Willmore functional to a wide range and some interesting results have been obtained.

In [12], Cai studied the $W_{(2, p)}$-Willmore functional for surfaces in 3-dimensional unit sphere $S^{3}(1)$ :

$$
W_{(2, p)}(\varphi)=\int_{M}\left(k_{1}-k_{2}\right)^{p} d v,
$$

where $k_{1}$ and $k_{2}$ denote the principal curvatures of $M$ and $k_{1} \geq k_{2}$. Under some proper conditions, Cai obtained some interesting inequalities. 
In [13], Guo and Li considered the $W_{(n, 1)}$-Willmore functional for submanifolds in unit sphere $S^{n+1}(1)$ :

$$
W_{(n, 1)}(\varphi)=\int_{M} \rho d v .
$$

They calculated the first variation and obtained Simons' type inequalities and finally classified the point-wise gap phenomenon. In [14], Xu and Yang derived the global but not point-wise gap phenomenon for the same functional.

In [15], Wu researched the $W_{(n, r)}$-Willmore functional for submanifolds in unit sphere $S^{n+1}(1)$ :

$$
W_{(n, r)}(\varphi)=\int_{M} \rho^{r} d v
$$

The first variation formula, Simons' type inequalities, and point-wise gap phenomenon are obtained.

Motivated by the work mentioned above, in this paper, we study an abstract Willmore type called $W_{(n, F)}$-Willmore functional:

$$
W_{(n, F)}(\varphi)=\int_{M} F(\rho) d v
$$

where we always assume that the abstract function satisfies

$$
F: u \longrightarrow F(u), \quad F \in C^{3}([0,+\infty) \longrightarrow R) .
$$

Obviously, functionals $W_{(n, n / 2)}, W_{(2, p)}, W_{(n, 1)}$, and $W_{(n, r)}$ are special cases of $W_{(n, F)}$, so the construction and investigation of $W_{(n, F)}$ are meaningful. As usual, the critical point of functional $W_{(n, F)}(\varphi)$ is called $W_{(n, F)}$-Willmore hypersurface. In Section 3, we calculate the 1st variation formula of functional $W_{(n, F)}$.

Theorem 1. Let $\varphi: M^{n} \rightarrow S^{n+1}(1)$ be an $n$-dimensional hypersurface in an $(n+1)$-dimensional unit sphere $S^{n+1}(1)$; then $M$ is a $W_{(n, F)}$-Willmore hypersurface if and only if

$$
\begin{aligned}
& \sum_{i j}\left(F^{\prime}(\rho) h_{i j}\right)_{, j i}-\Delta\left(F^{\prime}(\rho) H\right) \\
& \quad+\left(P_{3}-S H\right) F^{\prime}(\rho)-\frac{n}{2} F(\rho) H=0 .
\end{aligned}
$$

In particular, if $M$ is an isoparametric (all principal curvatures are constant) hypersurface, then $M$ is a $W_{(n, F)}$-Willmore hypersurface if and only if

$$
\left(P_{3}-S H\right) F^{\prime}(\rho)-\frac{n}{2} F(\rho) H=0 .
$$

Remark 2. when $F(\rho)=\rho^{n / 2}, \rho, \rho^{r}$, Theorem 1 coincides with corresponding results in $[6,13,15]$, respectively.

It is well known that Simons' integral inequality plays an important role in the study of minimal hypersurface. It says that if $M$ is an $n$-dimensional compact minimal hypersurface in $(n+1)$-dimensional unit sphere $S^{n+1}(1)$, then

$$
\int_{M} S(n-S) d v \leq 0
$$

For classic Willmore hypersurface, Li [6] proved a Simons' type integral result which says if $M$ is an $n$ dimensional compact classical Willmore hypersurface in unit sphere $S^{n+1}(1)$, then

$$
\int_{M} \rho^{n / 2}(n-\rho) d v \leq 0 .
$$

Similarly, for functionals $W_{(n, 1)}$ and $W_{(n, r)}$, $[13,15]$ obtained integral inequalities, respectively. A natural question is that whether a Simons' type inequality or equality can be established for $W_{(n, F)}$-Willmore functional. We give a confirm answer in this study.

Theorem 3. Let $M$ be an $n$-dimensional compact $W_{(n, F)^{-}}$ Willmore hypersurface in sphere $S^{n+1}(1)$; then we have Simons' type equality and can give a discussion according to the sign of $F, F^{\prime}$, and $F^{\prime \prime}$ :

$$
\begin{aligned}
0=\int_{M} & F^{\prime \prime}(\rho)|\nabla \rho|^{2}+2 F^{\prime}(\rho)\left(|\nabla h|^{2}-n|\nabla H|^{2}\right) \\
& -2 \rho(\rho-n) F^{\prime}(\rho)+n H^{2}\left(n F(\rho)-2 \rho F^{\prime}(\rho)\right) d v .
\end{aligned}
$$

(1) When $\rho \equiv n$, there holds

$$
\begin{aligned}
0=\int_{M} 2 F^{\prime}(n)\left(|\nabla h|^{2}-n|\nabla H|^{2}\right) \\
\\
\quad+n H^{2}\left(n F(n)-2 n F^{\prime}(n)\right) d v .
\end{aligned}
$$

(2) When $\rho \equiv 0$, there holds

$$
0=\int_{M} 2 F^{\prime}(0)\left(|\nabla h|^{2}-n|\nabla H|^{2}\right)+n^{2} H^{2} F(0) d v .
$$

(3) When $F^{\prime} \equiv 0, F \equiv c \neq 0$, there holds

$$
0=\int_{M} c n^{2} H^{2} d v
$$

(4) When $n F-2 u F^{\prime} \geq 0, F^{\prime} \geq 0, F^{\prime \prime} \geq 0$, there holds

$$
\begin{array}{rl}
\int_{M} & 2 \rho(\rho-n) F^{\prime}(\rho) \\
= & \int_{M} F^{\prime \prime}(\rho)|\nabla \rho|^{2}+2 F^{\prime}(\rho)\left(|\nabla h|^{2}-n|\nabla H|^{2}\right) \\
& +n H^{2}\left(n F(\rho)-2 \rho F^{\prime}(\rho)\right) d v .
\end{array}
$$

(5) When $n F-2 u F^{\prime} \leq 0, F^{\prime} \geq 0, F^{\prime \prime} \geq 0$, there holds

$$
\begin{array}{rl}
\int_{M} & 2 \rho(\rho-n) F^{\prime}(\rho)-n H^{2}\left(n F(\rho)-2 \rho F^{\prime}(\rho)\right) \\
= & \int_{M} F^{\prime \prime}(\rho)|\nabla \rho|^{2}+2 F^{\prime}(\rho)\left(|\nabla h|^{2}-n|\nabla H|^{2}\right) d v .
\end{array}
$$

Remark 4. When $F(\rho)=\rho^{n / 2}, \rho, \rho^{r}$, Theorem 3 coincides with corresponding results in $[6,13,15]$, respectively. Similar equalities also hold for the other sign cases of $F, F^{\prime}$, and $F^{\prime \prime}$.

Using the integral equalities in Theorem 3, together with two famous Chern et al. results in [16], we can obtain some conclusions of gap phenomenon. 
Theorem 5. Let $M$ be an n-dimensional closed $W_{(n, F)^{-}}$ Willmore hypersurface in unit sphere $S^{n+1}(1)$, we have the following.

(1) When $F^{\prime} \equiv 0, F \equiv c \neq 0$ over $(0,+\infty)$, if $0 \leq \rho \leq n$, then $\rho=0$ or $\rho=n$.

(a) For $\rho=0$, then $H=0, \rho=0, S=0$, and $M$ is totally geodesic;

(b) For $\rho=n$, then $H=0, \rho=n, S=n$, and $M=C_{m, n-m}=S^{m}(\sqrt{m / n}) \times S^{n-m}(\sqrt{(n-m) / n})$ for some $m$ with $1 \leq m \leq n-1$.

(2) When $n F-2 u F^{\prime}>0, F^{\prime}>0, F^{\prime \prime} \geq 0$ over $(0, \infty)$, if $0 \leq \rho \leq n$, then $\rho=0$ or $\rho=n$.

(a) For $\rho=0$, then $M$ is totally umbilical;

(b) For $\rho=n$, then $n \equiv 0(\bmod 2), H=0, \rho=n$, $S=n$, and $M=C_{n / 2, n / 2}=S^{n / 2}(\sqrt{1 / 2}) \times$ $S^{n / 2}(\sqrt{1 / 2})$.

(3) When $n F-2 u F^{\prime}=0, F^{\prime}>0, F^{\prime \prime} \geq 0$ over $(0,+\infty)$, if $0 \leq \rho \leq n$, then $F(u)=c u^{n / 2}, c>0, \rho=0$, or $\rho=n$.

(a) For $\rho=0, M$ is totally umbilical;

(b) For $\rho=n, M=W_{m, n-m}=S^{m}(\sqrt{(n-m) / n}) \times$ $S^{n-m}(\sqrt{m / n})$ for some $m$ with $1 \leq m \leq n-1$.

(4) When $n F-2 u F^{\prime}<0, F^{\prime}>0, F^{\prime \prime} \geq 0 \operatorname{over}(0,+\infty)$, if $H=0,0 \leq \rho \leq n$, then $H=0, \rho=0$ or $H=0, \rho=n$.

(a) For $H=0, \rho=0$, then $M$ is totally geodesic;

(b) For $H=0, \rho=n$, then $n \equiv 0(\bmod 2), H=0$, $\rho=n, S=n, M=C_{n / 2, n / 2}=S^{n / 2}(\sqrt{1 / 2}) \times$ $S^{n / 2}(\sqrt{1 / 2})$.

Remark 6. When $F(\rho)=\rho^{n / 2}, \rho, \rho^{r}$, Theorem 5 coincides with corresponding results in $[6,13,15]$, respectively. Similar conclusions also hold for the other sign cases of $F, F^{\prime}$, and $F^{\prime \prime}$. The examples appeared in Theorem 5 would be constructed in Section 3.

\section{Preliminaries}

Let $\varphi: M^{n} \rightarrow S^{n+1}(1)$ be an $n$-dimensional closed hypersurface in an $(n+1)$-dimensional unit sphere and let $\Phi(\cdot, \cdot): M \times(-\epsilon, \epsilon) \rightarrow S^{n+1}(1)$ be a variation of $\varphi$; it means that

$$
\varphi_{t}=: \Phi(\cdot, t): M \times\{t\} \longrightarrow S^{n+1}(1), \quad \forall t \in(-\epsilon, \epsilon)
$$

is still an isometric immersion with $\varphi_{0} \equiv \varphi$.

Let $s=\left(s_{1}, \ldots, s_{n}, s_{n+1}\right)$ and $\sigma=\left(\sigma^{1}, \ldots, \sigma^{n}, \sigma^{n+1}\right)$ be the orthonormal local frames of $T S^{n+1}(1)$ and $T^{*} S^{n+1}(1)$, respectively; then $e=\left(e_{1}, \ldots, e_{n}, e_{n+1}\right)=\varphi_{t}^{-1} s$ is the orthonormal local frames of the pullback vector bundle $\varphi_{t}^{-1} T S^{n+1}(1)$ over $M \times\{t\}$, such that $\left\{e_{1}, \ldots, e_{n}\right\}$ are tangent to $M$ and $\left\{e_{n+1}\right\}$ is normal to $M$ due to

$$
\varphi_{t}^{-1} T S^{n+1}(1)=T M_{t} \oplus T^{\perp} M_{t}
$$

Use $\omega$ to denote the connection form over $T S^{n+1}(1)$; by the pullback operation, we have the following decomposition:

$$
\begin{gathered}
\Phi^{*} \sigma=\theta+V d t, \quad \Phi^{*} \sigma^{i}=\theta^{i}+V^{i} d t, \\
\Phi^{*} \sigma^{n+1}=V^{n+1} d t=: f d t, \\
\Phi^{*} \omega=\phi+L d t, \quad \Phi^{*} \omega_{i}^{j}=\phi_{i}^{j}+L_{i}^{j} d t, \\
\Phi^{*} \omega_{i}^{n+1}=\phi_{i}^{n+1}+b_{i} d t .
\end{gathered}
$$

Thus, one can derive that $\left\{\theta^{i}\right\}$ are the orthonormal frames of $T^{*} M$ and $\theta^{n+1} \equiv 0$ when it is restricted over $M,\left\{V^{i}, V^{n+1}=\right.$ : $f\}$ are the variation vector fields of $\Phi,\left\{\phi_{i}^{j}\right\}$ is the connection form of $T M$, and $\left\{\phi_{i}^{n+1}=h_{i j} \theta^{j}\right\}$ is the second fundamental form. In particular, we have

$$
\left.\frac{\partial}{\partial t} \varphi_{t}\right|_{t=0}=\sum_{i} V^{i} e_{i}+f e_{n+1} .
$$

Use $\Omega$ and $\Omega^{\top}$ to denote curvature forms of $T S^{n+1}(1)$ and $T M$, respectively and write their components:

$$
\begin{gathered}
\Omega_{A B}=\frac{1}{2} \bar{R}_{A B C D} \sigma^{C} \wedge \sigma^{D}, \quad A, B, C, D \in\{1, \ldots, n+1\}, \\
\Omega_{i j}^{\top}=\frac{1}{2} R_{i j k l} \theta^{k} \wedge \theta^{l}, \quad i, j, k, l \in\{1,2, \ldots, n\} .
\end{gathered}
$$

From any one standard differential geometry book, we know that the curvature of unit sphere is

$$
\bar{R}_{A B C D}=-\left(\delta_{A C} \delta_{B D}-\delta_{A D} \delta_{B C}\right)
$$

Using connection form $\left\{\phi_{i}^{j}\right\}$, the covariant derivatives of $h_{i j}, H, \rho, V^{i}, f$, and $b_{i}$ can be defined as

$$
\begin{gathered}
h_{i j, k} \theta^{k}=d_{M} h_{i j}-\sum_{p} h_{p j} \phi_{i}^{p}-\sum_{p} h_{i p} \phi_{j}^{p}, \\
h_{i j, k l} \theta^{l}=d_{M} h_{i j, k}-\sum_{p} h_{p j, k} \phi_{i}^{p}-\sum_{p} h_{i p, k} \phi_{j}^{p}-\sum_{p} h_{i j, p} \phi_{k}^{p}, \\
H_{, i} \theta^{i}=d_{M} H, \quad H_{, i j} \theta^{j}=d_{M} H_{, i}-\sum_{p} H_{, p} \phi_{i}^{p},
\end{gathered}
$$




$$
\begin{gathered}
\Delta H=\sum_{i} H_{, i i}, \\
\rho_{, i} \theta^{i}=d_{M} \rho, \quad \rho_{, i j} \theta^{j}=d_{M} \rho_{, i}-\sum_{p} \rho_{, p} \phi_{i}^{p}, \quad \Delta \rho=\sum_{i} \rho_{, i i}, \\
V_{, j}^{i} \theta^{j}=d_{M} V^{i}+\sum_{p} V^{p} \phi_{p}^{i}, \\
V_{, j k}^{i} \theta^{k}=d_{M} V_{, j}^{i}-\sum_{p} V_{, p}^{i} \phi_{j}^{p}+\sum_{p} V_{, j}^{p} \phi_{p}^{i}, \\
f_{, i j} \theta^{j}=d_{M} f_{, i}-\sum_{p} f_{, p} \phi_{i}^{p}, \quad \Delta f=\sum f_{, i i}, \\
b_{i, j} \theta^{j}=d_{M} b_{i}-\sum_{p} b_{p} \phi_{i}^{p}, \\
b_{i, j k} \theta^{k}=d_{M} b_{i, j}-\sum_{p} b_{p, j} \phi_{i}^{p}-\sum_{p} b_{i, p} \phi_{j}^{p} .
\end{gathered}
$$

We use $d_{M}, d_{M \times(-\epsilon, \epsilon)}=d_{M}+d t(\partial / \partial t)$ and $d$ to denote the differential operators on $M, M \times(-\epsilon, \epsilon)$, and $S^{n+1}(1)$, respectively. By calculating directly and comparing both sides of the following equations:

$$
\begin{gathered}
\Omega=d \omega-\omega \wedge \omega, \quad 0=d \sigma-\sigma \wedge \omega, \\
\Phi^{*}(\Omega)=d_{M \times(-\epsilon, \epsilon)} \Phi^{*}(\omega)-\Phi^{*}(\omega) \wedge \Phi^{*}(\omega), \\
0=d_{M \times(-\epsilon, \epsilon)} \Phi^{*}(\sigma)-\Phi^{*}(\sigma) \wedge \Phi^{*}(\omega),
\end{gathered}
$$

we would derive out the lemmas below; also see article [10]. In fact, the right hand side of (27) is

$$
\begin{aligned}
\Phi^{*}\left(\Omega_{A}^{B}\right) & =\Phi^{*}\left(\frac{1}{2} \bar{R}_{A B C D} \sigma^{C} \wedge \sigma^{D}\right) \\
& =\frac{1}{2} \bar{R}_{A B C D} \Phi^{*}\left(\sigma^{C}\right) \wedge \Phi^{*}\left(\sigma^{D}\right) \\
& =\frac{1}{2} \bar{R}_{A B C D} \theta^{C} \wedge \theta^{D}+d t \wedge\left(\bar{R}_{A B C D} V^{C} \theta^{D}\right),
\end{aligned}
$$

while the left hand side of (27) is

$$
\begin{aligned}
d_{M \times(-\epsilon, \epsilon)} \Phi^{*}\left(\omega_{A}^{B}\right)-\Phi^{*}\left(\omega_{A}^{C}\right) \wedge \Phi^{*}\left(\omega_{C}^{B}\right) \\
=\left(d_{M}+d t \frac{\partial}{\partial t}\right)\left(\phi_{A}^{B}+d t L_{A}^{B}\right)-\left(\phi_{A}^{C}+d t L_{A}^{C}\right)\left(\phi_{C}^{B}+d t L_{C}^{B}\right) \\
=\left(d_{M} \phi_{A}^{B}-\phi_{A}^{C} \wedge \phi_{C}^{B}\right) \\
\quad+d t\left(\frac{\partial}{\partial t} \phi_{A}^{B}-\left(d_{M} L_{A}^{B}+L_{A}^{C} \phi_{C}^{B}-\phi_{A}^{C} L_{C}^{B}\right)\right) ;
\end{aligned}
$$

comparing (29) with (30), we obtain

$$
\begin{gathered}
\left(d_{M} \phi_{A}^{B}-\phi_{A}^{C} \wedge \phi_{C}^{B}\right)=\frac{1}{2} \bar{R}_{A B C D} \theta^{C} \wedge \theta^{D}, \\
\frac{\partial}{\partial t} \phi_{A}^{B}-\left(d_{M} L_{A}^{B}+L_{A}^{C} \phi_{C}^{B}-\phi_{A}^{C} L_{C}^{B}\right)=\bar{R}_{A B C D} V^{C} \theta^{D} .
\end{gathered}
$$

The equation of (28) is

$$
\begin{aligned}
0 & =d_{M \times(-\epsilon, \epsilon)} \Phi^{*}(\sigma)-\Phi^{*}(\sigma) \wedge \Phi^{*}(\omega) \\
& =\left(d_{M}+d t \frac{\partial}{\partial t}\right)(\theta+d t V)-(\theta+d t V) \wedge(\phi+d t L) \\
& =d_{M} \theta-\theta \wedge \phi+d t\left(\frac{\partial \theta}{\partial t}-d_{M} V-V \phi+\theta L\right) .
\end{aligned}
$$

Comparing both sides, we obtain

$$
d_{M} \theta-\theta \wedge \phi=0, \quad \frac{\partial \theta}{\partial t}-d_{M} V-V \phi+\theta L=0 .
$$

From (31) and (33), we can easily conclude the following lemmas which are useful in the variation calculation and establishment of Simons' type integral equalities.

Lemma 7. Let $\varphi: M^{n} \rightarrow S^{n+1}(1)$ be a hypersurface; one has structure equations:

$$
R_{i j k l}=-\left(\delta_{i k} \delta_{j l}-\delta_{i l} \delta_{j k}\right)-\left(h_{i k} h_{j l}-h_{i l} h_{j k}\right), \quad h_{i j, k}=h_{i k, j} .
$$

Lemma 8. Let $\varphi: M^{n} \rightarrow S^{n+1}(1)$ be a hypersurface; one has Ricci identity:

$$
\begin{aligned}
h_{i j, k l}-h_{i j, l k}= & -\left(\delta_{i k} h_{j l}-\delta_{i l} h_{j k}\right)-\left(\delta_{j k} h_{i l}-\delta_{j l} h_{i k}\right) \\
& -\left(h_{i k} h_{p l} h_{p j}-h_{i l} h_{p k} h_{p j}\right) \\
& -\left(h_{j k} h_{p l} h_{i p}-h_{j l} h_{p k} h_{i p}\right) .
\end{aligned}
$$

Lemma 9. Let $\varphi: M^{n} \rightarrow S^{n+1}(1)$ be a hypersurface and let $V=V^{\top}+V^{\perp}=V^{i} e_{i}+f e_{n+1}$ be a variation vector field; one has

$$
\begin{gathered}
\frac{\partial \theta^{i}}{\partial t}=\left(V_{, j}^{i}-L_{j}^{i}-h_{i j} f\right) \theta^{j}, \\
\frac{\partial h_{i j}}{\partial t}=f_{, i j}+\sum_{p} h_{i j, p} V^{p}+\sum_{p} h_{p j} L_{i}^{p}+\sum_{p} h_{i p} L_{j}^{p} \\
+\sum_{p} h_{i p} h_{p j} f+\delta_{i j} f .
\end{gathered}
$$

Lemma 10. With the same notations as above, one has

$$
\sum_{i j k} h_{i k} h_{k j} L_{j}^{i}=0, \quad \sum_{i j} h_{i j} L_{j}^{i}=0 .
$$

\section{Variation Calculation and Examples}

To calculate the first variation of $W_{(n, F)}$-Willmore functional, we need two lemmas.

Lemma 11. Let $\varphi: M^{n} \rightarrow S^{n+1}(1)$ be a hypersurface and let $V=V^{\top}+V^{\perp}=V^{i} e_{i}+f e_{n+1}$ be a variation vector field. Suppose that $d v=\theta^{1} \wedge \cdots \wedge \theta^{n}$ denotes the volume element; one has

$$
\frac{\partial d v}{\partial t}=\sum_{i} V_{, i}^{i}-n H f \text {. }
$$


Proof. By Lemma 9, we have $(\partial / \partial t)\left(\theta^{i}\right)=\left(V_{, j}^{i}-L_{j}^{i}-h_{i j} f\right) \theta^{j}$. Hence,

$$
\frac{\partial d v}{\partial t}=\sum_{i} \theta^{1} \wedge \cdots \frac{\partial}{\partial t}\left(\theta^{i}\right) \cdots \wedge \theta^{n}=\sum_{i} V_{, i}^{i}-n H f .
$$

With this, we complete the proof of Lemma 11.

Lemma 12. Let $\varphi: M^{n} \rightarrow S^{n+1}(1)$ be a hypersurface and let $V=V^{\top}+V^{\perp}=V^{i} e_{i}+f e_{n+1}$ be a variation vector field; one has

$$
\frac{\partial \rho}{\partial t}=\sum_{i j} 2 h_{i j} f_{, i j}-2 H \Delta f+2 P_{3} f-2 S H f+\sum_{i} \rho_{, i} V^{i}
$$

Proof. By the definition of $\rho, S$ and $H$, one has

$$
\frac{\partial}{\partial t}(\rho)=\frac{\partial}{\partial t}(S)-\frac{\partial}{\partial t}\left(n H^{2}\right)
$$

It follows Lemmas 9 and 10 that

$$
\begin{aligned}
\frac{\partial}{\partial t}(S)= & 2 \sum_{i j} h_{i j} \frac{\partial}{\partial t}\left(h_{i j}\right) \\
= & 2 \sum_{i j} h_{i j}\left[f_{, i j}+\sum_{p} h_{i j, p} V^{p}+\sum_{p} h_{p j} L_{i}^{p}\right. \\
& \left.+\sum_{p} h_{i p} L_{j}^{p}+\sum_{p} h_{i p} h_{p j} f+\delta_{i j} f\right] \\
= & 2 \sum_{i j} h_{i j} f_{, i j}+\sum_{i} S_{, i} V^{i}+2 P_{3} f+2 n H f .
\end{aligned}
$$

By Lemmas 9 and 10 again, one has

$$
\begin{aligned}
& \frac{\partial}{\partial t}\left(n H^{2}\right)= 2 n H \frac{\partial}{\partial t} H \\
&=2 H \sum_{i}\left[f_{, i i}+\sum_{p} h_{i i, p} V^{p}+\sum_{p} h_{p i} L_{i}^{p}\right. \\
& \\
&\left.\quad+\sum_{p} h_{p i} L_{i}^{p}+\sum_{p} h_{i p} h_{p i} f+\delta_{i i} f\right] \\
&=2 H \Delta f+\sum_{i}\left(n H^{2}\right)_{, i} V^{i}+2 H S f+2 n H f .
\end{aligned}
$$

Hence,

$$
\frac{\partial \rho}{\partial t}=\sum_{i j} 2 h_{i j} f_{, i j}-2 H \Delta f+2 P_{3} f-2 H S f+\sum_{i} \rho_{, i} V^{i} .
$$

With this, we complete the proof of Lemma 12.
Proof of Theorem 1. By Lemmas 11 and 12, we can calculate

$$
\begin{aligned}
& \frac{\partial}{\partial t} W_{(n, F)}\left(\varphi_{t}\right) \\
& =\int_{M_{t}} F^{\prime}(\rho) \frac{\partial}{\partial t}(\rho)+F(\rho)\left(\sum_{i} V_{, i}^{i}-n H f\right) d v \\
& =\int_{M_{t}} F^{\prime}(\rho)\left(\sum_{i j} 2 h_{i j} f_{, i j}-2 H \Delta f+2 P_{3} f-2 S H f+\sum_{i} \rho_{, i} V^{i}\right) \\
& +F(\rho)\left(\sum_{i} V_{, i}^{i}-n H f\right) d v \\
& =\int_{M_{t}}\left[\sum_{i j}\left(F^{\prime}(\rho) h_{i j}\right)_{, j i}-\Delta\left(F^{\prime}(\rho) H\right)\right. \\
& \left.+\left(P_{3}-S H\right) F^{\prime}(\rho)-\frac{n}{2} H F(\rho)\right] f d v .
\end{aligned}
$$

With this, we complete the proof of Theorem 1.

From Theorem 1, we can find a few examples in unit sphere $S^{n+1}(1)$, in particular, the isoparametric hypersurfaces. Since all principal curvatures $k_{1}, \ldots, k_{n}$ are constant, then $\rho, H, S$ are constant and $H=(1 / n) P_{1}, S=P_{2}, \rho=$ $P_{2}-(1 / n)\left(P_{1}\right)^{2}$. Thus, $W_{(n, F)}$-Willmore hypersurface equation becomes

$$
\left(P_{3}-\frac{1}{n} P_{1} P_{2}\right) F^{\prime}(\rho)-\frac{1}{2} P_{1} F(\rho)=0 .
$$

Example 13. Obviously, totally geodesic hypersurfaces are $W_{(n, F)}$-Willmore for any abstract function $F$, totally umbilical but not totally geodesic hypersurfaces are $W_{(n, F)}$-Willmore if and only if $F(0)=0$.

Example 14. For a particular hypersurface with $n \equiv 0(\bmod$ 2),

$$
C_{n / 2, n / 2}=S^{n / 2}\left(\frac{1}{\sqrt{2}}\right) \times S^{n / 2}\left(\frac{1}{\sqrt{2}}\right) \longrightarrow S^{n+1}(1) .
$$

All principal curvatures are

$$
k_{1}=\cdots=k_{n / 2}=1, \quad k_{(n / 2)+1}=\cdots=k_{n}=-1 .
$$

We can derive the quantities $P_{1}, P_{2}, P_{3}, \rho$, respectively, $P_{1}=$ $0, P_{2}=n, P_{3}=0, \rho=n$. Obviously $C_{n / 2, n / 2}$ always is a $W_{(n, F)^{-}}$ Willmore hypersurface of $S^{n+1}(1)$ for any function $F$.

Example 15. For a family hypersurfaces with parameters $\lambda, \mu, 0<\lambda, \mu<1, \lambda^{2}+\mu^{2}=1$.

$$
S^{m}(\lambda) \times S^{n-m}(\mu) \longrightarrow S^{n+1}(1), \quad 1 \leq m \leq n-1 .
$$

Obviously, all principal curvatures are

$$
k_{1}=\cdots=k_{m}=\frac{\mu}{\lambda}, \quad k_{m+1}=\cdots=k_{n}=-\frac{\lambda}{\mu} .
$$


Then the quantities $P_{1}, P_{2}, P_{3}, \rho$ are, respectively,

$$
\begin{gathered}
P_{1}=m \frac{\mu}{\lambda}-(n-m) \frac{\lambda}{\mu}, \quad P_{2}=m \frac{\mu^{2}}{\lambda^{2}}+(n-m) \frac{\lambda^{2}}{\mu^{2}} \\
P_{3}=m \frac{\mu^{3}}{\lambda^{3}}-(n-m) \frac{\lambda^{3}}{\mu^{3}} \\
\rho=\frac{m(n-m)}{n}\left[\frac{\mu^{2}}{\lambda^{2}}+\frac{\lambda^{2}}{\mu^{2}}+2\right] .
\end{gathered}
$$

Setting $\mu / \lambda=x>0, W_{(n, F)}$-Willmore hypersurface equation becomes

$$
\begin{aligned}
& 2 m(n-m) F^{\prime}(\rho) x^{6}-m\left[n F(\rho)-2(n-m) F^{\prime}(\rho)\right] x^{4} \\
& \quad+(n-m)\left[n F(\rho)-2 m F^{\prime}(\rho)\right] x^{2} \\
& \quad-2 m(n-m) F^{\prime}(\rho)=0 .
\end{aligned}
$$

Example 16 (Clifford torus $[16] ; F(\rho) \equiv 1)$. Consider that

$$
C_{m, n-m}=S^{m}\left(\sqrt{\frac{m}{n}}\right) \times S^{n-m}\left(\sqrt{\frac{n-m}{n}}\right), \quad 1 \leq m \leq n-1
$$

are minimal hypersurfaces with $H \equiv 0, S \equiv n, \rho \equiv n$.

Example 17 (classic Willmore torus [6]; $F(\rho)=\rho^{n / 2}$ ). Consider that

$$
W_{m, n-m}=S^{m}\left(\sqrt{\frac{n-m}{n}}\right) \times S^{n-m}\left(\sqrt{\frac{m}{n}}\right), \quad 1 \leq m \leq n-1
$$

are $W_{(n, n / 2)}$-Willmore hypersurfaces with $\rho=n$. When some $W_{m, n-m}$ is minimal, then $n \equiv 0(\bmod 2), m=n / 2$.

\section{Simons' Type Equalities and \\ Gap Phenomenon}

Some lemmas are needed for the establishment of Simons' type integral equalities and the discussion of gap phenomenon.

Lemma 18. For $\rho$, one has

$$
\begin{aligned}
\Delta \rho= & \sum_{i j} 2 n h_{i j} H_{, i j}-2 n H \Delta H+2|\nabla h|^{2} \\
& -2 n|\nabla H|^{2}+2 n \rho+2 n H P_{3}-2 S^{2},
\end{aligned}
$$

where $|\nabla h|^{2}=\sum_{i j k} h_{i j, k}^{2}$.
Proof. By the definition of $\rho$ and Laplacian operator, we have

$$
\begin{aligned}
\Delta \rho & =\sum_{k}\left(\sum_{i j} h_{i j}^{2}-n H^{2}\right)_{, k k} \\
& =\sum_{k}\left(\sum_{i j} 2 h_{i j} h_{i j, k}-2 n H H_{, k}\right)_{, k} \\
& =\sum_{k}\left(\sum_{i j} 2 h_{i j, k}^{2}+\sum_{i j} 2 h_{i j} h_{i j, k k}-2 n H_{, k}^{2}-2 n H H_{, k k}\right) \\
& \left.=2|\nabla h|^{2}-2 n H \Delta H-2 n|\nabla H|^{2}+2 \sum_{i j k} h_{i j} h_{i j, k k}\right) \\
& =2\left(|\nabla h|^{2}-n H \Delta H-n|\nabla H|^{2}+\left(T_{1}\right) \sum_{i j k} h_{i j} h_{i j, k k}\right),
\end{aligned}
$$

for term $T_{1}=\sum_{i j k} h_{i j} h_{i j, k k}$; by Lemmas 7 and 8 , easily we obtain

$$
\begin{aligned}
T_{1} & =\sum_{i j k} h_{i j} h_{i j, k k}=\sum_{i j k} h_{i j} h_{i k, j k} \\
& =\sum_{i j k} h_{i j}\left(h_{i k, j k}-h_{i k, k j}+h_{i k, k j}\right) \\
& =\sum_{i j k} h_{i j} h_{k k, i j}+\sum_{i j k} h_{i j}\left(h_{i k, j k}-h_{i k, k j}\right) \\
& =\sum_{i j} n h_{i j} H_{, i j}+\left(T_{2}\right) \sum_{i j k} h_{i j}\left(h_{i k, j k}-h_{i k, k j}\right),
\end{aligned}
$$

for term $T_{2}=\sum_{i j k} h_{i j}\left(h_{i k, j k}-h_{i k, k j}\right)$; by Lemma 8, we have $T_{2}$

$$
\begin{aligned}
= & \sum_{i j k} h_{i j}\left(h_{i k, j k}-h_{i k, k j}\right) \\
= & \sum_{i j k} h_{i j}\left\{\left[-\left(\delta_{j k} h_{i k}-\delta_{k k} h_{i j}\right)-\left(h_{j k} h_{i p} h_{p k}-h_{k k} h_{i p} h_{p j}\right)\right]\right. \\
& \left.+\left[-\left(\delta_{i j} h_{k k}-\delta_{i k} h_{j k}\right)-\left(h_{i j} h_{k p} h_{p k}-h_{i k} h_{k p} h_{p j}\right)\right]\right\} \\
= & -S+n S-\sum_{i j k p} h_{i j} h_{j k} h_{i p} h_{p k}+n H P_{3}-n^{2} H^{2} \\
& +S-S^{2}+\sum_{i j k p} h_{i j} h_{i k} h_{k p} h_{p j} \\
= & n \rho-S^{2}+n H P_{3} .
\end{aligned}
$$

Substituting (58) into (57), we have

$$
T_{1}=\sum_{i j} n h_{i j} H_{, i j}+n \rho-S^{2}+n H P_{3} .
$$


Substituting (59) into (56), we have

$$
\begin{array}{rl}
\Delta \rho=\sum_{i j} & 2 n h_{i j} H_{, i j}-2 n H \Delta H+2|\nabla h|^{2} \\
& \quad-2 n|\nabla H|^{2}+2 n \rho+2 n H P_{3}-2 S^{2} .
\end{array}
$$

With this, we complete the proof of Lemma 18.

Lemma 19 (Huisken's estimate [17]). Consider that

$$
|\nabla h|^{2} \geq \frac{3 n^{2}}{n+2}|\nabla H|^{2} \geq n|\nabla H|^{2},
$$

and $|\nabla h|^{2}=n|\nabla H|^{2}$ if and only if $\nabla h=0$.

Proof. We decompose the tensor $\nabla h$ as follows:

$$
h_{i j, k}=E_{i j k}+F_{i j k} \text {, }
$$

where

$$
\begin{gathered}
E_{i j k}=\frac{n}{n+2}\left(H_{, i} \delta_{j k}+H_{, j} \delta_{i k}+H_{, k} \delta_{i j}\right), \\
F_{i j k}=h_{i j, k}-E_{i j k} .
\end{gathered}
$$

Through a computation, we obtain

$$
|E|^{2}=\frac{3 n^{2}}{n+2}|\nabla H|^{2}, \quad\langle E, F\rangle=0 .
$$

Finally, by the triangle inequality, we have

$$
|\nabla h|^{2} \geq|E|^{2}=\frac{3 n^{2}}{n+2}|\nabla H|^{2} \geq n|\nabla H|^{2} .
$$

If $|\nabla h|^{2}=n|\nabla H|^{2}$, then all inequalities become equalities, $F_{i j k}=0, \nabla H=0$, and $E_{i j k}=0$; thus, $\nabla h=0$. With this, we complete the proof of Lemma 19.

Lemma 20. For $F(\rho)$, one has

$$
\begin{aligned}
\Delta F(\rho)= & F^{\prime \prime}(\rho)|\nabla \rho|^{2}+2 F^{\prime}(\rho)\left(|\nabla h|^{2}-n|\nabla H|^{2}\right) \\
& -2 \rho(\rho-n) F^{\prime}(\rho)+n H^{2}\left(n F(\rho)-2 \rho F^{\prime}(\rho)\right) \\
& +2 n\left[\sum_{i j} F^{\prime}(\rho) h_{i j} H_{, i j}-F^{\prime}(\rho) H \Delta H\right. \\
& \left.+\left(P_{3}-S H\right) F^{\prime}(\rho) H-\frac{n}{2} F(\rho) H^{2}\right] .
\end{aligned}
$$

Proof. We know that

$$
\Delta F(\rho)=F^{\prime \prime}(\rho)|\nabla \rho|^{2}+F^{\prime}(\rho) \Delta \rho
$$

By Lemmas 18 and 19, we can easily obtain the identity.
Proof of Theorem 3. Integrating the equality in Lemma 20 over $M$, we have

$$
\begin{array}{r}
0=\int_{M} F^{\prime \prime}(\rho)|\nabla \rho|^{2}+2 F^{\prime}(\rho)\left(|\nabla h|^{2}-n|\nabla H|^{2}\right) \\
-2 \rho(\rho-n) F^{\prime}(\rho)+n H^{2}\left(n F(\rho)-2 \rho F^{\prime}(\rho)\right) \\
+2 n\left[\sum_{i j} F^{\prime}(\rho) h_{i j} H_{, i j}-F^{\prime}(\rho) H \Delta H\right. \\
\left.+\left(P_{3}-S H\right) F^{\prime}(\rho) H-\frac{n}{2} F(\rho) H^{2}\right] .
\end{array}
$$

By Theorem 1, we know that

$$
\begin{aligned}
\sum_{i j} & \left(F^{\prime}(\rho) h_{i j}\right)_{, j i}-\Delta\left(F^{\prime}(\rho) H\right) \\
& +\left(P_{3}-S H\right) F^{\prime}(\rho)-\frac{n}{2} F(\rho) H=0 .
\end{aligned}
$$

Using Stokes theorem, we obtain

$$
\begin{aligned}
& 0=\int_{M} F^{\prime \prime}(\rho)|\nabla \rho|^{2}+2 F^{\prime}(\rho)\left(|\nabla h|^{2}-n|\nabla H|^{2}\right) \\
&-2 \rho(\rho-n) F^{\prime}(\rho)+n H^{2}\left(n F(\rho)-2 \rho F^{\prime}(\rho)\right) .
\end{aligned}
$$

We complete the proof of Theorem 3.

In order to prove Theorem 5, we need two very important conclusions which are treated as a lemma and a main Theorem in Chern et al.s article [16]. For a hypersurface, we choose frame fields in such a way that $h_{i j}=0$, for all $i \neq j, h_{i}=h_{i i}$.

Lemma 21 (Lemma 3 of [16]). Let $x: M^{n} \rightarrow S^{n+1}(1)$ be a compact hypersurface with $\nabla h \equiv 0$; then there are two cases.

(1) $h_{1}=\cdots=h_{n}=\lambda=$ constant, and $M$ is a totally umbilic $(\lambda>0)$ or totally geodesic $(\lambda=0)$;

(2) $h_{1}=\cdots h_{m}=\lambda=$ constant $>0, h_{m+1}=\cdots=h_{n}=$ $-(1 / \lambda), 1 \leq m \leq n-1$, and $M$ is a Riemannian product of $M_{1} \times M_{2}$, where $M_{1}=S^{m}\left(1 / \sqrt{1+\lambda^{2}}\right), M_{2}=$ $S^{n-m}\left(\lambda / \sqrt{1+\lambda^{2}}\right)$.

Lemma 22 (main Theorem of [16]). Clifford torus $C_{m, n-m}, 1 \leq$ $m \leq n-1$ are the only compact minimal $(H=0)$ hypersurfaces of dimension $n$ in unit sphere $S^{n+1}(1)$ satisfying $S=n$.

Proof of Theorem 5. Obviously, case (1) of Theorem 5 is a corollary of Lemma 22; thus, we just focus cases (2) and (3); the rest can be proved by similar argument. 
(2) When $n F-2 u F^{\prime}>0, F^{\prime}>0, F^{\prime \prime} \geq 0$ over $(0,+\infty)$, by the case (4) of Theorem 3 , we have

$$
\begin{array}{rl}
\int_{M} & 2 \rho(\rho-n) F^{\prime}(\rho) \\
=\int_{M} F^{\prime \prime}(\rho)|\nabla \rho|^{2}+2 F^{\prime}(\rho)\left(|\nabla h|^{2}-n|\nabla H|^{2}\right) \\
\quad+n H^{2}\left(n F(\rho)-2 \rho F^{\prime}(\rho)\right) \geq 0
\end{array}
$$

If $0 \leq \rho \leq n$, the right hand side of identity (71) is nonpositive, while the left hand side of (71) is nonnegative; thus, we can conclude that $\rho=0$ or $\rho=n$. For $\rho=0, M$ is totally umbilical; for $\rho=n$, substituting it into the above equality together with Lemma 19, we can derive $\nabla h=0, H=0, \rho=n$, and $S=n$, by Lemma 22, we know that $M=C_{m, n-m}$; moreover, by $W_{(n, F)^{-}}$ Willmore equation, we conclude that $n \equiv 0(\bmod 2), H=0$, $\rho=n, S=n$, and $M=C_{n / 2, n / 2}$.

(3) When $n F-2 u F^{\prime}=0, F^{\prime}>0, F^{\prime \prime} \geq 0(0,+\infty)$, we know that $F(u)=c u^{n / 2}, c=$ const $>0$. By the case $(4)$ of Theorem 3, we have

$$
\begin{aligned}
& \int_{M} \rho^{n / 2}(\rho-n) \\
& \quad=\int_{M} \frac{n-2}{4}|\nabla \rho|^{2}+\rho^{(n / 2)-1}\left(|\nabla h|^{2}-n|\nabla H|^{2}\right) \geq 0 .
\end{aligned}
$$

Moreover, if $0 \leq \rho \leq n$, then $\rho=0$ or $\rho=n$. For $\rho=0$, $M$ is totally umbilical; for $\rho=n$, substituting it into the above equality together with Lemma 19 , we obtain $\nabla h=0$; by Lemma 22, we know that $M$ is a torus; then, by Example 17, we can conclude that $M$ is a classic Willmore torus $W_{m, n-m}$ for some $m$.

\section{Conflict of Interests}

The authors declare that there is no conflict of interests regarding the publication of this paper.

\section{Acknowledgment}

The second author would like to express thanks to Professor H. Z. Li for his encouragement and help.

\section{References}

[1] B. Y. Chen, "Some conformal invariants of submanifolds and their applications," Bollettino della Unione Matematica Italiana, vol. 10, pp. 380-385, 1974.

[2] F. J. Pedit and T. J. Willmore, "Conformal geometry," Atti del Seminario Matematico e Fisico dell'Università di Modena, vol. 36, pp. 237-245, 1988.

[3] F. J. Willmore, Total Curvature in Riemannian Geometry, Ellis Horwood, 1982.

[4] T. J. Willmore, Riemannian Geometry, Oxford Science Publications, Clarendon Press, Oxford, UK, 1993.

[5] F. C. Marques and A. Neves, "Min-max theory and the Willmore conjecture," Annals of Mathematics, vol. 179, no. 2, pp. 683-782, 2014.
[6] H. Z. Li, "Willmore hypersurfaces in a sphere," Asian Journal of Mathematics, vol. 5, pp. 365-378, 2001.

[7] H. Z. Li, "Willmore surfaces in Sn," Annals of Global Analysis and Geometry, vol. 21, no. 2, pp. 203-213, 2002.

[8] H. Z. Li, "Willmore submanifolds in a sphere," Mathematical Research Letters, vol. 9, no. 5-6, pp. 771-790, 2002.

[9] Z. Guo, "Willmore submanifolds in the unit sphere," Collectanea Mathematica, vol. 55, no. 3, pp. 279-287, 2004.

[10] Z. J. Hu and H. Z. Li, "Willmore submanifolds in Riemannian manifolds," in Proceedings of the Workshop on Contemporary Geometry and Related Topics, pp. 251-275, World Scientific, Hackensack, NJ, USA, 2005.

[11] Z. Guo, H. Z. Li, and C. P. Wang, "The second variation of formula for Willmore submanifolds in Sn," Results in Mathematics, vol. 40, pp. 205-225, 2001.

[12] M. Cai, " $L^{p}$ willmore functionals," Proceedings of the American Mathematical Society, vol. 127, no. 2, pp. 569-575, 1999.

[13] Z. Guo and H. Z. Li, "A variational problem for submanifolds in a sphere," Monatshefte für Mathematik, vol. 152, no. 4, pp. 295302, 2007.

[14] H. W. Xu and D. Y. Yang, "The gap phenomenon for extremal submanifolds in a sphere," Differential Geometry and its Application, vol. 29, no. 1, pp. 26-34, 2011.

[15] L. Wu, "A class of variational problems for submanifolds in a space form," Houston Journal of Mathematics, vol. 35, no. 2, pp. 435-450, 2009.

[16] S. S. Chern, M. do Carmo, and S. Kobayashi, "Minimal submanifolds of a sphere with second fundamental form of constant length," in Functional Analysis and Related Fields, pp. 59-75, Springer, Berlin, Germany, 1970.

[17] G. Huisken, "Flow by mean curvature of convex surfaces in to shperes," Journal of Differential Geometry, vol. 20, pp. 237-266, 1984. 


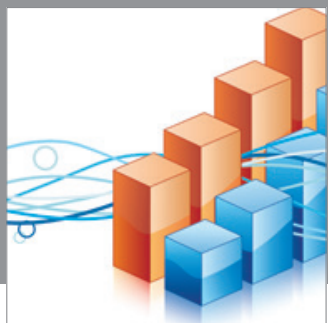

Advances in

Operations Research

mansans

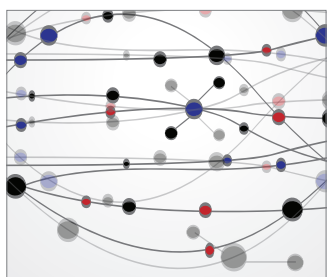

The Scientific World Journal
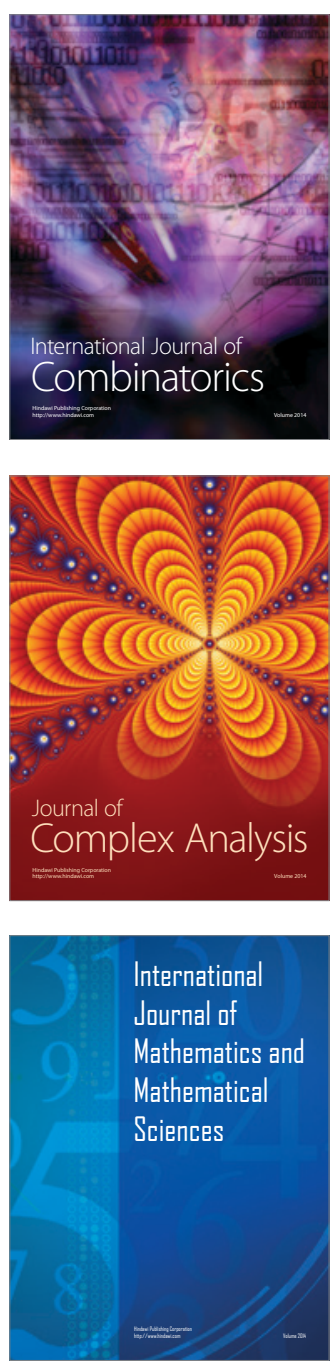
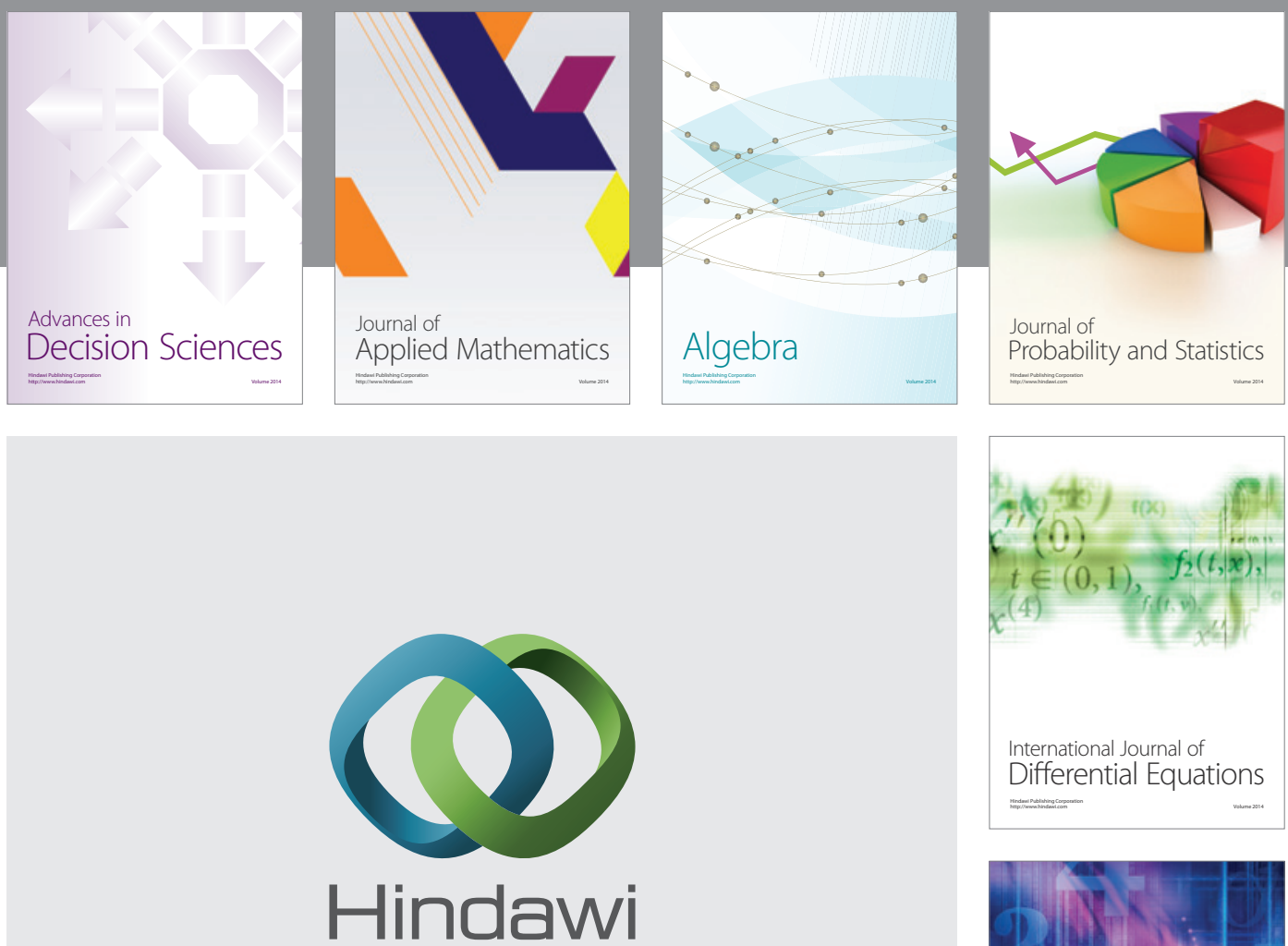

Submit your manuscripts at http://www.hindawi.com
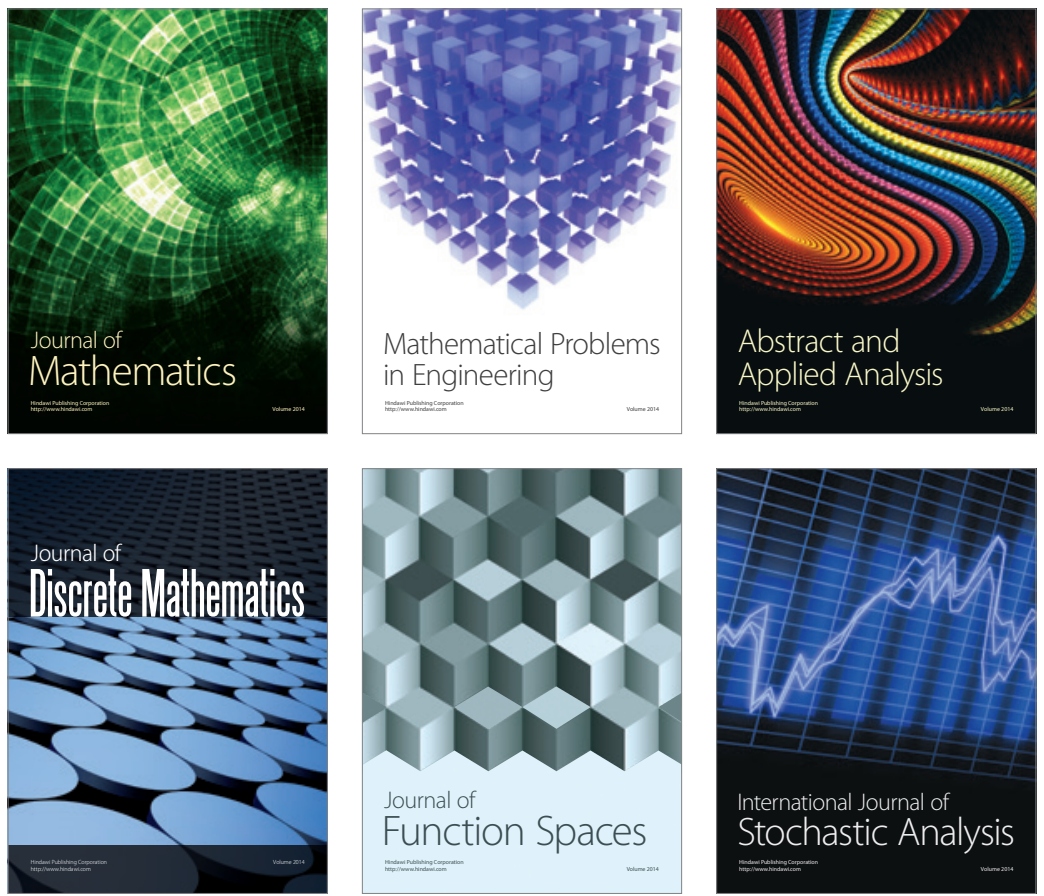

Journal of

Function Spaces

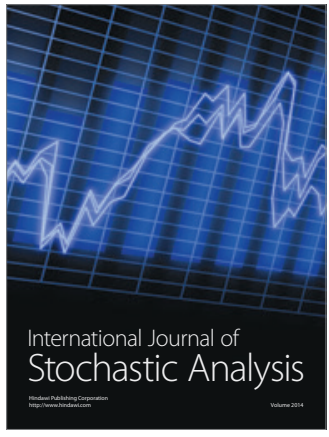

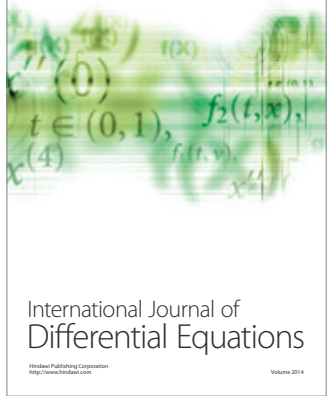
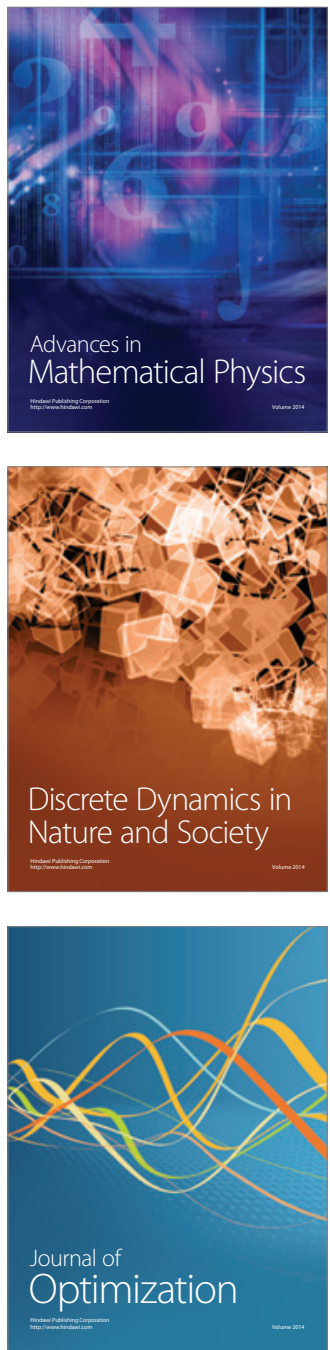